\title{
eJRIEPS
}

Ejournal de la recherche sur l'intervention en éducation physique et sport

12 | 2007

Varia

\section{Vers une rénovation de la situation traditionnelle de conseil pédagogique}

Sébastien Chaliès, Eric Flavier et Stéfano Bertone

\section{(2) OpenEdition}

\section{Journals}

Édition électronique

URL : https://journals.openedition.org/ejrieps/6154

DOI : 10.4000/ejrieps.6154

ISSN : 2105-0821

Éditeur

ELLIADD

\section{Référence électronique}

Sébastien Chaliès, Eric Flavier et Stéfano Bertone, « Vers une rénovation de la situation traditionnelle de conseil pédagogique », eJRIEPS [En ligne], 12 | 2007, mis en ligne le 01 juillet 2007, consulté le 28 juin 2021. URL : http://journals.openedition.org/ejrieps/6154 ; DOI : https://doi.org/10.4000/ejrieps. 6154

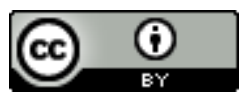

La revue eJRIEPS est mise à disposition selon les termes de la Creative Commons Attribution 4.0 International License. 
Sébastien Chaliès* ${ }^{*}$ Eric Flavier ${ }^{* *}$ \& Stéfano Bertone ${ }^{* * *}$

* DATIEF (ERTe n`60 - IUFM de Nice) - IUFM de Toulouse, France.

** DATIEF (ERTe n60 - IUFM de Nice) - IUFM de Strasbourg, France.

*** DATIEF (ERTe n60 - IUFM de Nice) - IUFM de Nice, France.

\section{Résumé}

L'objet de cette étude était d'apprécier les effets potentiels d'une situation collaborative de conseil pédagogique sur le développement professionnel d'enseignants novices. Le développement professionnel a été étudié en utilisant une approche herméneutique et inductive basée sur l'analyse de données issues de l'observation et d'entretiens d'autoconfrontation. En analysant dans le détail la qualité de l'articulation entre les situations traditionnelles de formation (situations de co-préparation ou de co-bilan de la leçon) et la situation de classe, les résultats précisent les effets de la situation collaborative de conseil pédagogique sur la formation professionnelle des enseignants novices. Les conditions d'une utilisation de ces résultats pour optimiser les dispositifs de formation sont finalement discutées.

\section{Introduction}

L'objet de cette étude de cas était d'apprécier les effets potentiels d'un aménagement de la situation traditionnelle de conseil pédagogique par un renforcement de la collaboration entre les enseignants novices (EN) et leur conseiller pédagogique (CP).

L'efficacité de la situation de conseil pédagogique est régulièrement discutée (voir pour une revue Chaliès \& Durand, 2000). Certaines études montrent ainsi que la valorisation du soutien émotionnel des EN par les CP se fait au détriment d'un accompagnement réflexif efficace (Stanulis \& Russel, 2000). Ce manque d'efficacité est lié au dilemme que vivent les $\mathrm{CP}$ : prescrire aux EN des solutions prêtes à l'emploi à court terme et/ou s'engager dans la durée avec eux dans la construction de ces solutions (Bertone, Chaliès, Clarke \& Méard, 2006 ; Orland-Barak, 2005). Dans ces situations d'interaction les CP ont 
conscience de leur responsabilité et éprouvent des sentiments intenses de culpabilité ou de compassion à l'égard des EN (Bullough \& Draper, 2004). Ils s'engagent alors souvent dans une pratique réflexive à la place des EN plutôt qu'à leurs côtés (Loughran \& Berry, 2005). Sans véritable formation sur les "learning conversations" (Orland, 2000), les CP interagissent avec les EN de façon beaucoup plus prescriptive et contrôlée qu'ils ne le pensent (Beck \& Kosnik, 2000) et manquent de nombreuses opportunités de formation qui s'offrent à eux au cours de l'entretien (Carver \& Katz, 2004). Ces difficultés sont d'autant plus importantes que les $\mathrm{CP}$, les $\mathrm{EN}$ et les formateurs universitaires (FU) ont du mal à s'inscrire de façon cohérente dans le dispositif de formation par alternance entre les "two largely separate worlds" (Beck \& Kosnik, 2000) que sont l'université et l'établissement scolaire (Bullough \& Draper, 2004).

Pour répondre à ces insuffisances, certains auteurs préconisent de renouveler le modèle traditionnel de conseil pédagogique en instaurant a "highly interactive learning community" (Paris \& Gespass, 2001) ou "a collaborative mentoring model" (Mullen, 2000) impliquant les EN, les CP et les FU dans "a process of co-constructing supervision" (Paris \& Gespass, 2001). Cette proposition est intéressante car elle permet à chacun des acteurs, d'une part, de négocier le travail à accomplir à partir de ses propres préoccupations, attentes et possibilités (Awaya, McEwan, Heyler, Linsky, Lum \& Wakukawa, 2003; Paris \& Gespass, 2001) et, d'autre part, de se développer professionnellement (Grisham, Ferguson \& Brink, 2004) en s'impliquant véritablement lors des temps de pratique réflexive (Weiss \& Weiss, 2001) et professionnelle (Burbank \& Kauchak, 2003). Impliqués dans une relation plus collaborative, les EN et leur CP construisent "a creative and democratic space" permettant confiance, partage et entraide (Jipson \& Paley, 2000). Epaulés par les $\mathrm{CP}$, les EN s'impliquent alors davantage dans leur pratique professionnelle en prenant plus de risques en classe (Stanulis \& Russell, 2000), et dans les entretiens post-leçon lors desquels les demandes d'aide sont rendues possibles (Eick, Ware \& Williams, 2003). 
La plupart de ces études portent sur les effets d'une situation collaborative de conseil pédagogique sur la formation pour se centrer en priorité sur la qualité de la situation d'entretien post-leçon. Elles notent, par exemple, l'amélioration chez les EN de leur pratique réflexive (Parsons \& Stephenson, 2005), ou encore de leur construction de connaissances professionnelles lorsqu'ils collaborent davantage avec les CP (Jenkins \& Vael, 2004). Moins nombreuses, d'autres études discutent des effets d'une situation collaborative de conseil pédagogique sur le travail d'enseignement des EN. L'engagement des EN auprès de leurs élèves devient alors plus "risqué" (Burbank \& Kauchak, 2003) ou caractérisé de "prises d'initiatives" (Bullough et al., 2003).

Toutefois, aucune étude à notre connaissance n'a cherché à apprécier la qualité de l'articulation entre des situations traditionnelles de formation (entretiens de conception et d'analyse du travail à réaliser ou réalisé lors de la leçon) et des situations de classe lors de la réalisation d'une situation collaborative de conseil pédagogique. Aucune étude ne précise, par exemple, l'impact de l'amélioration de la pratique réflexive des EN ou encore de leurs connaissances professionnelles sur leur activité en classe. L'objectif de cette étude était donc d'apprécier ces effets.

\section{Cadre théorique}

Appartenant à un programme plus large, cette étude s'inscrit directement dans le cadrage théorique de l'équipe de recherche «Développement de l'Activité, Travail, Identité des Enseignants en Formation » de l'IUFM de Nice. Elle s'appuie, plus précisément, sur une conceptualisation de la formation professionnelle à partir de postulats empruntés : (a) à la psychologie culturaliste de Vygotski (1978) et, (b) à la philosophie analytique de Wittgenstein (1996; 2004).

(a) Selon une approche culturaliste, l'apprentissage du métier se réalise au cours de situations de formation. Le plus souvent menées sous la forme de dialogues (comme par exemple les entretiens de préparation ou de bilan de la leçon), ces situations engendrent 
un développement de l'activité professionnelle des EN par les concepts et les instruments (Vygotski, 1978) co-construits avec les CP ou les FU au moyen d'une analyse du travail réalisé en classe. C'est à cette occasion que les EN se construisent des "instruments psychologiques" nouveaux (Vygotski, 1978) avec l'aide des CP ou des FU. Ces instruments alimentent ensuite l'activité décisionnelle des EN en situation de classe. Ils leur permettent notamment d'anticiper les évènements dans la classe, de percevoir et de donner du sens à des événements nouveaux ou encore d'envisager des actions à réaliser pour s'y adapter (Bertone, Méard, Ria, Euzet \& Durand, 2003).

(b) Ces instruments psychologiques construits lors de situations de formation peuvent être assimilées à des "règles constitutives du métier" (Wittgenstein, 1996) qui sont des ressources permettant aux EN de construire la signification de contextes nouveaux d'enseignement et d'action. L'apprentissage de ces règles suppose la construction d'un accord entre les EN et les CP établissant des "liens de signification" (Wittgenstein, 1996) entre une expérience de classe considérée comme exemplaire par les CP et la façon dont ces derniers ont l'habitude de la concevoir et de la désigner au plan professionnel. A partir des règles apprises, les EN s'attendent potentiellement à percevoir ces situations remarquables que les $\mathrm{CP}$ leur ont adressées en formation. Les règles apprises constituent alors pour les EN une sorte de "mesure-étalon" permettant de juger, interpréter et/ou agir correctement, c'est-à-dire conformément aux règles (Wittgenstein, 1996, 2004).

\section{Méthode}

\subsection{Participants :}

Un EN PLC2 EPS de l'IUFM de Midi-Pyrénées et son CP ont été volontaires pour participer à cette étude. L'EN avait une expérience en enseignement de sept semaines réparties au cours des deux années précédentes de formation. II avait pour la première fois la responsabilité de deux classes pendant une année scolaire complète. Le CP était à 
la fois considéré comme un enseignant d’EPS expérimenté (12 années d'expérience, membre du «Groupe Ressource » de l'Académie et à ce titre participation comme formateur dans le cadre du Plan Académique de Formation) mais aussi comme un conseiller pédagogique expérimenté (Six années d'expérience dans cette fonction, suivi à plusieurs reprises du stage de formation à la fonction de conseiller pédagogique proposé par l'IUFM). L'observation a été réalisée lors de leçons de badminton avec une classe de 24 élèves de 4 ème.

\subsection{Dispositif :}

La situation traditionnelle de conseil pédagogique a été aménagée pour réaliser cette étude à partir des lignes directrices de l'"educative mentoring" (Feiman Nemser, 2001).

La situation collaborative de conseil pédagogique proposée à l'EN et au CP était constituée de quatre étapes successives réparties sur deux semaines consécutives du troisième trimestre de l'année scolaire 2004-2005 : i) le CP a observé la leçon de l'EN; ii) à la fin de la leçon, l'EN et le CP ont fait un bilan puis planifié la leçon suivante (Situation de co-préparation de la leçon); iii) cette leçon a été conduite par l'EN et le CP auprès des élèves de l'EN la semaine suivante (Situation de co-intervention); iv) à la fin de la leçon, l'EN et le CP ont réalisé un bilan (Situation de co-bilan).

\subsection{Recueil des données}

Deux catégories de données ont été recueillies.

Les données d'enregistrement ont été recueillies pendant les quatre étapes composant la situation collaborative de conseil pédagogique. Une caméra vidéo ainsi que deux micro HF portés par l'EN et le CP ont été utilisés pour faire ce recueil.

Les données d'autoconfrontation ont été recueillies à partir d'un enregistrement audiovidéo des entretiens d'autoconfrontation menés par le chercheur $(C)$ avec chacun des acteurs à l'issue de chacun des quatre temps du dispositif de formation. Le C a conduit ces entretiens afin de connaître les jugements portés par l'enseignant interviewé à propos 
de ses actions ou des actions d'autrui visionnées. Ses questions incitaient l'enseignant interviewé à décrire et justifier ses jugements (par exemple : "Qu'est ce que tu penses de cette remarque?"; " Cette action te parait-elle satisfaisante ?"; " Es-tu en accord ou en désaccord avec ces propos ou cette façon de faire?"). Le C relançait régulièrement son questionnement soit en demandant de préciser le jugement préalablement porté (par exemple: "Je ne comprends pas pourquoi tu considères cette action comme intéressante, peux-tu reprendre ?») soit en intervenant sur le mode de la controverse en plaçant l'enseignant interviewé face à des contradictions apparentes (par exemple : "tu dis que les élèves sont plus calmes mais aussi pas forcément plus attentifs, peux tu préciser?"). Finalement, le $\mathrm{C}$ cherchait par ce questionnement à recueillir le plus grand nombre d'éléments pour reconstituer a posteriori les règles apprises par les enseignants et utilisées pour réaliser leurs jugements.

\subsection{Analyse des données}

L'objet de cette analyse était de situer l'utilisation des expériences de formation lors de l'expérience de classe ou inversement. Pour ce faire, nous avons cherché à identifier les règles de métier adressées par le CP et utilisées par l'EN en formation ou lors de la réalisation du travail.

L'analyse des données a été effectuée en trois étapes successives.

Etape 1 : Après la retranscription des données d'observation et d'autoconfrontation, le corpus a été découpé en unités d'interaction. Ces unités ont été délimitées à partir de l'objet des jugements portés par les acteurs lors des entretiens d'autoconfrontation. Une nouvelle unité d'interaction a été créée à chaque fois que l'objet du jugement porté par les acteurs changeait.

Etape 2 : Pour chaque unité d'interaction, le jugement porté par les acteurs et l'étayage de ce jugement ont été identifiés. L'étayage du jugement a été associé à l'ensemble des éléments évoqués par le locuteur pour « expliquer au chercheur la façon 
de s'y prendre pour juger, de la même façon et avec la même règle que lui-même, une action professionnelle ou un événement de la classe ». Un débat critique a été engagé à chaque fois que possible à propos de la désignation des événements / actions en question et des jugements formulés.

Étape 3 : Pour chaque unité d'interaction, la règle utilisée par l'acteur pour porter son jugement a été formalisée. Chaque règle a été étiquetée à partir : i) de l'objet du jugement porté, et ii) des éléments d'étayage de ce jugement. Dans sa forme, chaque règle a été présentée ainsi : ["Objet" vaut pour "éléments d'étayage"]. Afin de minimiser les interprétations du $\mathrm{C}$, chaque règle a été étiquetée à partir du vocabulaire des acteurs.

\subsection{Validité des données}

La procédure de validation s'est déroulée en deux temps. Tout d'abord, l'ensemble du corpus a été analysé de façon séparée par chaque $C$ afin d'identifier et de formaliser : i) les unités d'interaction, ii) les jugements portés et leur étayage, iii) les règles utilisées pour porter les jugements. Les résultats obtenus ont ensuite fait l'objet d'une analyse commune. Ils ont été comparés et discutés par les $C$ jusqu'à l'obtention d'un accord. Sur l'ensemble du corpus analysé, moins de $5 \%$ des éléments identifiés ont finalement été source de désaccord et rejetés.

\section{Résultats}

L'aménagement de la situation traditionnelle de conseil pédagogique par un renforcement de la collaboration avec le CP a eu des effets sur la formation professionnelle de l'EN (Tableau I). 
Tableau I. Règles de métier apprises et/ou suivies par l'enseignant novice lors des situations de co-préparation, de co-intervention et de co-bilan avec le conseiller pédagogique.

\begin{tabular}{lccc}
\hline & \multicolumn{3}{c}{ Règles de métier } \\
\cline { 2 - 4 } $\begin{array}{l}\text { Situations } \\
\text { dispositif }\end{array}$ & Suivies & Apprises & Total \\
\hline Co-préparation & 10 & 4 & 14 \\
Co-intervention & 17 & 4 & 21 \\
Co-bilan & 12 & 1 & 13 \\
Total & 39 & 9 & 48 \\
\hline
\end{tabular}

Nous avons identifiés 48 règles de métier apprises (19\%) et/ou suivies (81\%) par l'EN sur l'ensemble du dispositif. L'EN a appris de nouvelles règles de métier lors de chacune des temps du dispositif. Proportionnellement, des différences apparaissent toutefois entre les temps. L'EN a davantage appris lors de la situation de co-préparation $(28.5 \%$ des règles identifiées pour cette situation), puis lors de la situation de co-intervention (19\% des règles identifiées pour cette situation) et enfin de la situation de co-bilan $(7.5 \%$ des règles identifiées pour cette situation).

Sur l'ensemble de ces règles de métier apprises, seules deux ont été suivies par l'EN au cours du dispositif. Notre objet d'étude étant d'apprécier la qualité de l'articulation entre les situations de formation (Situations de co-préparation ou Situation de co-bilan de la leçon) et les situations de classe (Situation de co-intervention) lors de la réalisation d'une situation collaborative de conseil pédagogique nous avons fait le choix, pour cet article, d'analyser dans le détail l'apprentissage et le suivi d'une de ces règles par l'EN.

Lors de la leçon en co-intervention, l'EN a exploité une règle de métier apprise lors de la situation de co-préparation réalisée antérieurement avec le CP. A cet instant de la leçon, l'EN et le CP se sont placés dans un coin du gymnase pour observer ensemble les élèves au travail. Ils ont jugé que ces derniers ne réalisaient pas ce qui leur avait été demandé (marquer le point en frappant le volant selon un mouvement vers le bas) et ont échangé à 
propos des raisons de cette difficulté et des actions à réaliser pour les résoudre (Extrait 1) :

\section{Extrait 1:}

EN: Je pense qu'il faut revenir sur [L'EN mime l'action de frappe du volant permettant de le rabattre vers le sol], parce qu'ils [les élèves] sont tous... Comme ça [Le CP mime l'action de frappe du volant des élèves orientée vers le plafond], tu sais?

CP : Ouais ...

EN: Ils [les élèves] frappent par en dessous, comme ça par en dessous [L'EN mime l'action de frappe du volant des élèves orientée vers le plafond]...

$C P$ : Ouais, il faut qu'on leur fasse une démonstration!

EN : Ouais... Avec, on prend le volant devant soi et on casse bien le poignet pour rabattre vers le bas [le CP mime le geste tout en le verbalisant]... CP : Oui...

Lors de l'entretien d'autoconfrontation, l'EN a justifié son action auprès du CP. L'extrait qui suit permet d'identifier la règle de métier que l'EN a utilisée lors de la leçon pour s'engager dans l'interaction avec le CP (Extrait 2).

\section{Extrait 2 :}

EN : Et là je répète, du coup... je répète avec lui [le CP] les éléments là, à reprendre... J'essaye presque de me mettre en situation là.

$C:$ Tu répètes avec lui [le $C P]$, c'est-à-dire ?

EN : Je fais la liste [des actions à réaliser] pour rien oublier... Casser le poignet...

Enfin quelque part là, je sens que je vais devoir le dire donc...

$C$ : D’accord! Là, en fait, tu, tu essayes de préparer ce que tu vas dire [aux élèves] ?

EN : Ouais, en quelque sorte!

$C$ : Et donc tu me dis, je répète... Tu peux expliciter un peu ça là ?

EN : Presque comme si je lui [au CP] expliquais... J'essaye de verbaliser, de bien clarifier ce que je veux dire... Pour après pouvoir aller le donner aux élèves... Et c'est un truc [verbaliser les opérations à réaliser avant de les donner aux élèves] qu'il [le CP] fait lui aussi quand on prépare ensemble... II l'a fait [lors de la copréparation de la leçon]... 
$C:$ C'est à dire?

EN : Sur l'entretien qu'on a la semaine d'avant... Quand on préparait [la leçon] il [le CP] l'a, il les a dites [les actions à réaliser] mais comme si, comme s'il parlait aux élèves...

L'EN a délivré au C des éléments d'étayage permettant de comprendre son usage de la règle énoncée : "se préparer à énoncer les actions à réaliser aux élèves" nécessite de se placer "en situation" de présentation des opérations à réaliser comme si l'on s'adressait déjà aux élèves. II a cherché à le faire auprès du CP en "essayant de verbaliser", "de répéter avec lui", de "faire la liste" des éléments à délivrer, "comme s'il lui expliquait". En suivant cette règle de métier, l'EN s'attendait à "bien clarifier" et à "ne pas oublier" d'informations à énoncer aux élèves lors de la démonstration.

L'Extrait 2 permet aussi d'accéder à l'histoire des usages de cette règle de métier par l'EN, le $\mathrm{CP}$ et le $\mathrm{C}$. Cette règle a été tout d'abord adressée par le CP à l'EN lors de la copréparation de la leçon. Elle a ensuite été suivie par l'EN lors de la situation de classe. Formulée par le C, elle a enfin été utilisée par l'EN pour interpréter a posteriori ses actions de classe. L'extrait d'entretien d'autoconfrontation proposé ci-après et relatif à l'entretien de co-préparation de la leçon avec le CP permet de renforcer l'idée selon laquelle l'apprentissage de cette règle de métier par l'EN a eu lieu lors de l'entretien de copréparation de la leçon (Extrait 3).

\section{Extrait 3 :}

EN : Là je l'écoute [le CP]. Il construit la leçon directement là...Et là je suis en train de penser qu'il est en train... Dans sa tête, quand il [le CP] prépare la situation, il formule déjà la consigne [les opérations à réaliser] qu'il va donner aux élèves...

\section{C : Tu peux revenir sur ça ? Qu'est-ce qu'il fait là ?}

EN : Il est en train de dérouler la situation là, déjà... En fait, il prépare... En fait, dans sa façon de préparer il gagne du temps et surtout il est... II ne rate rien, il n'oublie rien sur le... Une fois sur le terrain. Parce qu'en la préparant, il va dire... On va faire... On va les mettre [les élèves] deux par terrain et puis on va faire travailler la rupture... Et il va plus loin que ça... II dit « je sers, l'autre renvoie... », là il est déjà en train de parler aux élèves... Comme s'il parlait aux élèves déjà.

$C$ : Donc toi à ce moment là ? 
EN : Là je comprends là !

C : Le contenu?

EN : Non la manière de préparer et d'être clair une fois sur le terrain... En fait, il répète son texte... Il a déjà le texte préparé et il déroule... C'est surtout le fait qu'il formule déjà la consigne avant de la donner aux élèves...

L'Extrait 3 permet d'apprécier la qualité de l'articulation entre une situation de formation de type co-préparation de la leçon et une situation de classe lors de la réalisation d'une situation collaborative de conseil pédagogique. II montre que c'est à l'occasion de la situation de co-préparation de la leçon que l'EN a "compris quelle est la manière de préparer [la leçon] pour être clair une fois sur le terrain [auprès des élèves]". II a alors appris en observant les actions du CP une règle de métier qu'il a ensuite exploitée lors de la leçon en co-intervention. L'EN a donc construit un lien de signification entre une expérience qu'il a considérée comme exemplaire ("là je suis en train de penser qu'il est en train... Dans sa tête, quand il [le CP] prépare la situation, il formule déjà la consigne qu'il va donner aux élèves...", Extrait 3) et une règle d'action qui a été à la fois un moyen d'élaboration de son travail et une mesure étalon pour interpréter ses expériences en classe ("Et là, je répète du coup...Je répète avec lui [le CP] les éléments là, à reprendre... J'essaye presque de me mettre en situation là...Presque comme si je lui expliquais... J'essaye de verbaliser, de bien clarifier ce que je veux dire... Pour après pouvoir aller le donner aux élèves", Extrait 2).

Outre cette règle de métier apprise lors de la situation de co-préparation, l'EN suit aussi lors de la leçon en co-intervention une règle de métier apprise par observation des actions menées par le CP au sein même de la situation de co-intervention. Finalement, même si les situations de co-préparation ou de co-bilan semblent particulièrement propices à la formation de l'EN, la situation de co-intervention y participe aussi. Cette situation permet à la fois l'apprentissage de nouvelles règles par l'EN par observation de ce que fait le CP 
mais aussi et surtout leur exploitation immédiate dans des circonstances identiques de réalisation du métier.

\section{Discussion et conclusion}

Cette étude montre la participation d'une situation collaborative de conseil pédagogique au décloisonnement des situations de co-préparation ou de co-bilan, traditionnellement consacrées à la formation, par rapport au travail d'enseignement proprement dit. Elle présente l'impact des interactions de formation sur le développement professionnel de l'EN en situation de classe. Une circonstance particulière s'est toutefois révélée favorable à ce développement. Cette étude montre, en effet, que l'apprentissage des règles de métier a lieu lors de situations où l'EN a été en mesure de se préparer à agir comme son CP. Ainsi par exemple, les actions du CP réalisées en situation de classe, celles relatives à la conception des instructions à délivrer aux élèves ou bien encore celles relatives à l'analyse des difficultés des élèves en classe ont été observées et utilisées par l'EN comme ressources pour interpréter autrement la situation de classe et agir auprès des élèves. Ces résultats sont en accord avec les conclusions d'autres études relevant l'intérêt pour l'EN d'observer le CP en situation de classe (Eick et al, 2003; Orland-Barak, 2005; Stanulis \& Russell, 2000) et non plus seulement dans les situations traditionnelles de formation. En donnant à voir à l'EN ce qu'il fait dans une situation d'enseignement similaire, le CP est plus directif mais aussi et surtout plus explicite (Feiman-Nemser, 2001; Loughran \& Berry, 2005).

Un résultat spécifique de cette étude concerne le constat qu'en situation collaborative de conseil pédagogique l'EN se construit la possibilité de percevoir et de s'attendre à des événements non perçus auparavant. Les propositions apprises par exemple en copréparation avec le CP ont en effet réorganisé en profondeur la signification que l'EN a attribuée aux situations professionnelles vécues par la suite. L'observation des actions 
professionnelles réalisées par le $\mathrm{CP}$, et la construction en co-intervention de liens de signification nouveaux peuvent aussi être considérées comme des sources d'apprentissage situé de règles de métier par l'EN.

Les expériences de métier jugées comme exemplaires appréhendées sous ces modalités d'interaction de formation jouent le rôle de véritables "mesures-étalons" (Wittgenstein, 1996) pour l'EN, lui permettant à la fois d'agir et de porter des jugements différents sur ses propres expériences de classe. Il en a été de même concernant la construction d'attentes nouvelles par l'EN. Lors de la co-conception des instructions à délivrer aux élèves, par exemple, l'EN a construit des attentes en imaginant un contexte fictif de classe. L'aide apportée par le CP à la réalisation de cette opération a consisté à énoncer les instructions « comme si » elles étaient déjà adressées aux élèves. A l'occasion de la situation de copréparation de la leçon l'EN a compris quelle était la manière de préparer la leçon pour être clair auprès des élèves. II en a été de même lors des interactions à propos de l'analyse des difficultés des élèves en classe. Les énoncés de l'EN en classe ont permis de constater la construction d'attentes nouvelles et l'élargissement de la signification des règles apprises lors de l'expérience de formation au cours des interactions situées avec les élèves.

Les résultats de cette étude permettent également de préciser que la transformation de l'expérience de formation au cours de l'expérience de classe a été étroitement liée à la présence du CP comme destinataire, au même titre que les élèves, des actions de l'EN dans la classe. Outre le support émotionnel qu'elle procure (Eick et al., 2003 ) et les possibilités d'apprentissage qu'elle ouvre (Roth, Masciotra \& Boyd, 1999), la présence du CP au sein de la situation de classe est une aide au développement professionnel de l'EN. II est possible de concevoir la présence du CP comme une sorte de « destinataire de secours » (Todorov, 1981) ayant incité l'EN à appliquer les règles préalablement apprises, comme autant de mesures-étalons pour juger l'expérience de la classe présente. Plus 
précisément cette présence aurait permis aux actions professionnelles réalisées ou énoncées par le CP de devenir des instruments psychologiques intra-psychiques (Vygotski, 1978) pour l'EN. La présence du CP comme destinataire des actions de l'EN en classe est donc apparue comme une circonstance facilitant l'élargissement de la signification des règles apprises en formation et l'augmentation du pouvoir d'action de I'EN.

Les résultats de cette étude renforcent finalement l'idée que l'activité décisionnelle de l'EN en classe est caractérisée par l'évocation d'actions possibles à réaliser en rapport avec les interlocuteurs (destinataires) présents et significatifs dans la situation (Bertone et al., 2003). Ils permettent aussi de spécifier la qualité de ces destinataires et accordent une place importante au caractère participatif des actions réalisées et des décisions prises en situation de classe. En effet, les règles apprises en formation par l'EN ne sont pas réalisées en classe lorsque la formation porte uniquement sur une co-analyse de l'expérience vécue et que le CP n'est présent que comme observateur lors de la leçon suivant la formation (Bertone et al., 2003). Les dispositifs à haut potentiel de formation professionnelle sont donc ceux qui optimisent l'accompagnement de l'EN au sein même de la leçon en valorisant la mise en œuvre d'une activité de co-intervention avec le CP.

\section{Bibliographie}

Awaya, A., McEwan, H., Heyler, D., Linsky, S., Lum, D., Wakukawa, P.(2003). Mentoring as a journey. Teaching and Teacher Education, 19(1), 45-56.

Beck, C., Kosnik, C. (2000). Associate teachers in pre-service education : Clarifying and enhancing their roles. Journal of Education for Teaching, 26(3), 208-224.

Bertone, S., Méard, J.A., Ria, L., Euzet, J.P., Durand, M. (2003). Intrapsychic conflict experienced by a preservice teacher during classroom interactions: A case study in physical education. Teaching and Teacher Education, 19(1): 113-125.

Bertone, S., Chaliès, S., Clarke, A., Méard, J.A. (2006). The dynamics of interaction during post-lesson conferences and the development of professional activity: Study of a 
preservice physical education teacher and her cooperating teacher. Asian Pacific Journal of Teacher Education.

Bullough, R. V., Draper, R. J. (2004). Mentoring and the emotions. Journal of Education for Teaching, 30(3), 271-288.

Bullough, J. Young, J., Birrell, J., Clark, C., Winston Egan, M., Erickson, L., Frankovich, M., Brunetti, J., Welling, M. (2003). Teaching with a peer : A comparison of two models of student teaching. Teaching and Teacher Education, 19, 57-73.

Burbank, M.D., Kauchak, D. (2003). An alternative model for professionnal development :investigations into effective collaboration. Teaching and Teacher Education, 19, 499-514.

Carver, C.L., Katz, D.S. (2004). Teaching at the boundary of acceptable practice : What is a new teacher mentor to do ? Journal of Teacher Education, 55(5), 449-462.

Chaliès, S., Durand, M. (2000). L'utilité discutée du tutorat en formation initiale des enseignants. Recherche et Formation, 35, 145-180.

Eick, C., Ware, F., Williams, P. (2003). Co-teaching in a science methods course : A situated learning model of becoming a teacher. Journal of Teacher Education, 54(1), 74-85.

Feiman-Nemser, S. (2001). Helping novices learn to teach : Lessons from an exemplary support teacher. Journal of Teacher Education, 52(1), 17-30.

Grisham, D.L, Ferguson, J.L., Brink, B. (2004). Mentoring the mentors: Student teachers'contributions to the middle school classroom. Mentoring and Tutoring. 12(3), 307-319.

Jenkins, J.M., Vael, M.L. (2004). Preservice teachers'PCK development during peer coaching. Journal of Teaching in Physical Education. 22(1), 20-28.

Jipson, J., Paley, N. (2000). Because no one gets there alone : collaboration as comentoring. Theory into practice, 39(1), 36 - 42.

Loughran, J., Berry, A. (2005). Modelling by teacher educators. Teaching and Teacher Education, 21, 193-203.

Mullen, C. (2000). Constructing co-mentoring partnership : walkways we must travel. Theory into practice, 39(1), 4-11.

Orland, L. (2000). Reading a mentoring situation: One aspect of learning to mentor. Teaching and Teacher Education, 17, 75-88.

Orland-Barak, L. (2005). Cracks in the iceberg: Surfacing the tensions of constructivist pedagogy. Teachers and Teaching: Theory and Practice, 11(3), 293-313. 
Paris, C., Gespass, S. (2001). Examining the mismatch between learner-centered teaching and teacher-centered supervision. Journal of Teacher Education, 52(5), 398-412.

Parsons, M., Stephenson, M. (2005). Developping reflective practice in student teachers : Collaboration and critical partnerships. Teachers and Teaching: Theory and Practice, 11(1), 95-116.

Roth, W., Masciotra, D., Boyd, N. (1999). Becoming in the classroom. A case study of teacher development through coteaching. Teaching and Teacher Education, 15, 771-784.

Stanulis, R. N., Russell, D. (2000). "jumping in" : Trust and communication in mentoring student teachers. Teaching and Teacher Education, 16, 65-80.

Todorov, T. (1981). Mikhaïl Bakhtine : le principe dialogique. Paris : Seuil.

Vygotski, L. S. (1978). Mind in society: The development of higher mental processes. Cambridge, MA: Harvard University Press.

Weiss, E. M., Weiss, S. (2001). Doing reflective supervision with student teachers in a Professional Development School Culture. Reflective Practice, 2(2), 125-154.

Wittgenstein, L. (1996). Remarques philosophiques. Oxford, Blackwell : G.E.M. Anscomb et G.H. Von Wright (Eds.).

Wittgenstein, L. (2004). De la certitude. Oxford, Blackwell : G.E.M. Anscomb et G.H. Von Wright (Eds.). 